\title{
Nuclear medicine 2013: from status quo to status go
}

\author{
Thomas Beyer • Marcus Hacker • August Schubiger • \\ Irene Virgolini • Hans-Jürgen Wester
}

Received: 29 August 2013/Accepted: 30 August 2013 / Published online: 3 October 2013

(C) Springer-Verlag Berlin Heidelberg 2013

For decades nuclear medicine has been fostered as a selfsustaining medical monoculture. Since the 1950s, when focusing on the thyroid gland, nuclear medicine physicians were confident in embracing a holistic approach to the diagnosis and therapy of thyroid diseases. At the time, radionuclide therapy was introduced with the clinical adoption of ${ }^{131}$ I-iodide for the diagnosis and therapy of differentiated thyroid carcinoma. This early theranostic concept reflects a prime example of the inherent capabilities of nuclear medicine: to use the same biomolecule both for assessing the extent of a disease and for

This editorial represents the essence of the discussions during the 8th Tyrolean Nuclear Medicine Symposium on 28 June 2013 in Innsbruck, Austria, held in honour of the 80th birthday of Prof. Georg Riccabona.

T. Beyer $(\square)$

Center for Medical Physics and Biomedical Engineering, General

Hospital Vienna, Medical University of Vienna, Waehringer Guertel

18-20/4L, A-1090 Vienna, Austria

e-mail: thomas.beyer@meduniwien.ac.at

\section{Hacker}

Department of Biomedical Imaging and Image-Guided Therapy, Division of Nuclear Medicine, Medical University of Vienna,

Vienna, Austria

T. Beyer $\cdot$ M. Hacker

Research Cluster Imaging, Medical University of Vienna, Vienna, Austria

\section{A. Schubiger \\ Collegium Helveticum, Transdisciplinary Laboratory, ETH and UZH \\ Zurich, Zurich, Switzerland}

\section{Virgolini}

Department of Nuclear Medicine, Medical University Innsbruck, Innsbruck, Austria

\section{H.-J. Wester}

Pharmaceutical Radiochemistry, Technical University Munich, Garching, Germany subsequent image-guided therapy, requiring only its labelling with a different radioisotope.

In 1991, a comprehensive review of radiotherapeutics was published [1]. This review described about 40 substances that appeared to be promising probes for various disease pathways. Since then only few therapeutic drugs have been developed and adopted for routine use: ${ }^{131}$ I-Iodide and ${ }^{131} \mathrm{I}$-MIBG, and another ten radiopharmaceuticals based on radiolabelled phosphonates and $\mathrm{Ca}^{2+}$ analogues (for the treatment of bone metastases) or ${ }^{90} \mathrm{Y}$-particles/microspheres (for the treatment of liver metastases). While several radiolabelled antibodies and the first somatostatin analogue were also presented in that early paper [1], it was only in 2012 that the FDA approved of the first ${ }^{90}$ Y-radiolabelled antibody (Zevalin) [2]. Today, in 2013, first somatostatin analogues $\left({ }^{111} \mathrm{In} /{ }^{90} \mathrm{Y}\right.$-octreotide and ${ }^{68} \mathrm{Ga} /{ }^{177} \mathrm{Lu}-T y{ }^{3}$-octreotate) are in clinical phase III trials.

Also, from an imaging perspective, nuclear medicine has a lot to add to the management of patients. Nuclear medicine imaging techniques, such as SPECT and PET, are instrumental in revealing causes and pathways of a variety of diseases. The clinical success of $\left[{ }^{18} \mathrm{~F}\right]$ FDG PET, in particular, has resulted in the wider acceptance of nuclear medicine imaging, and PET in particular, as a means to provide biological signals as an integral part of disease management. Likewise, the availability of a number of SPECT tracers has supported the adoption of nuclear medicine imaging in diagnostic pathways, such as those in dementia, cardiology and oncology [3]. The success story of PET, and to a somewhat lesser degree of SPECT, is also based on the hardware combination of these modalities with CT.

Being fully aware of many of the obstacles to nuclear medicine dissemination, as early as the late 1990s nuclear medicine experts conceived the idea of anatometabolic imaging [4] by means of dual modality imaging systems [5]. In addition to its clinical benefits [6], dual modality imaging offers a number of methodological benefits [7, 8]. Dual modality imaging has further supported the transition towards 
new detector materials, such as LYSO for PET and solid-state detectors (CZT) for SPECT imaging, both of which offer vastly increased system performance and completely revised system designs [9]. Thus, dual modality imaging has not only been inspired by nuclear medicine, but it has brought nuclear medicine to a new level of performance.

The most important prerequisite for the integrated, imagebased diagnosis-and-treatment path, mentioned at the beginning, is the cooperation of physicians, radiopharmacists and physicists. Such multidisciplinary cooperation is inherent to nuclear medicine. However, nuclear medicine experts do not necessarily extend this cooperation beyond their own field of expertise. The frequently observed inability to communicate the strengths of our specialty, the lack of open-minded discussions of the limitations of our specialty, or the dislike of commenting on either, are major roadblocks to strengthening nuclear medicine techniques today and to their use in clinical and research applications in the future. Together, we have reached a status quo of nuclear medicine, where we, nuclear medicine friends and specialists, feel confident of the advantages of our specialty, but are not good at promoting them beyond our own field.

Looking back at the progress made in tracer development, there have been countless minor successes but few breakthroughs. The commitment of the pharmaceutical industry in this field during the past decade has been sporadic and has resulted mainly in the development and approval of agents for early imaging of Alzheimer's disease. In this situation, academic research will likely remain the driving force for the development of most new radiopharmaceuticals. As a consequence, academic research centres will continue to advance in-house approaches, even if this is only the alteration of the substitution pattern of a promising tracer developed by another group. Such an isolated approach may become more popular than an interinstitutional, translational approach aiming to evaluate, to select and to subsequently transfer promising new tracers into multicentre clinical settings, which should be the overarching objective for nuclear medicine in clinical disease management.

On the other end of nuclear medicine, imaging, by means of SPECT/CT and PET/CT, for example, is supported clinically and methodologically by a number of expert advocates in nuclear medicine. However, many of these advocates are preaching to the converted and only a few of them present results from their professional specialty to an audience that is made up typically of radiology-avid experts. Obviously, wider dissemination of nuclear medicine applications at the current time is also limited by the economic circumstances, the lack or reduction of reimbursement in general, and illogical requirements to prove the value and efficacy of nuclear medicine techniques by means of randomized controlled trials [10], a concept that has not been applied equally to radiological imaging examinations.

Furthermore, a close look at both imaging modalities reveals a largely inefficient adoption of SPECT/CT and PET/CT across the globe. A recent survey among SPECT/CT users
[11] found that the majority of examinations are performed with little attention to the CT element. Likewise, few users of $\mathrm{PET} / \mathrm{CT}$ utilize the full potential of the CT element through the use of radiology-equivalent protocols [12]. This is in addition to a general variability in adhering to imaging standards [13]. Furthermore, either a dual certified radiologist and nuclear medicine expert, or both together, report only $30 \%$ of combined imaging examinations. It is therefore not surprising that neither dual modality is recognized widely in clinical standards.

Likewise, the recently introduced combined and even fully integrated PET and MRI can be seen as an advance in nuclear medicine [14]. While the technological hurdles are much higher than for the integration of SPECT or PET and CT, the potential benefits in terms of extracting multiple layers of information are also higher [15]. PET/MRI would further elevate nuclear medicine applications by helping to extract multiparametric data. Such multiparametric data analysis will benefit and could even be linked to the development and availability of powerful imaging probes, as mentioned above.

Unfortunately, many early adopters of integrated PET/MRI seem to be unimpressed by past experience, or to quote from Sir Bernhard Shaw (1856-1950): "If history repeats itself and the unexpected always happen, how incapable must Man be of learning from experience?". Shortly after the clinical introduction of PET/MRI, behind that of PET/CT by a decade, studies were published that promoted either the use of low-quality MRI for anatomical landmarking of the PET data or hailed the improved alignment of the bladder on simultaneously acquired PET and MR images. These studies investigated the low-hanging fruit to pick during the early phase following the introduction of this new imaging modality. However, they missed the point of translating the potential of this impressive yet expensive imaging combination [16].

All of the examples above illustrate the widespread ability of nuclear medicine to support an integrated diagnostic and therapeutic treatment path. However, major obstacles within and around our specialty exist and need to be recognized and urgently addressed. So, which activities are required to prepare nuclear medicine for the future, to increase the clinical acceptance of nuclear medicine applications leading to their widespread use? Where should nuclear medicine go from here?

We propose a five-point action plan:

1. Together we need to increase awareness of the full spectrum of nuclear medicine techniques. This includes active communication and cooperation with other medical disciplines and associations, much like the participation in lobbying efforts. We need to foster collaborative activities between oncologists, neurologists, cardiologists and others with nuclear medicine specialists to support efforts for better patient care. On the methodological side we need to strive to see nuclear medicine data formats (e.g. 
listmode, dynamic data, SUV, fusion images) included in the PACS environment as well as in remote data viewing and analysis platforms.

2. Together we need to present nuclear medicine data as fully quantitative and reproducible information for individualized image-guided therapy planning and monitoring. This includes in-vivo tissue characterization through multiple available radiotracers and the exploration of new therapy response markers and the cross-validation and integration of nuclear medicine parameters with imaging biomarkers of other modalities, such as MRI and CT. For this purpose, we need to work more closely with basic molecular medicine disciplines, such as histopathology and molecular genetics.

3. Together we need to significantly promote and advance the development and production of clinically relevant radiopharmaceuticals. This includes the implementation of production environments that are acceptable in light of good manufacturing regulations which are being continually tightened and applied [17]. Promising tracers should be evaluated in concerted interinstitutional and comparative studies at an early stage of development [18]. A valid selection would help to subsequently focus on joint multicentre clinical studies suitable for preparing applications for marketing authorizations in a stepwise manner.

4. Together we need to establish a state-of-the-art curriculum as the basis for the next generation of medical professionals. Such a curriculum should reflect the objective of strengthening nuclear medicine, preferably as an independent clinical discipline. Such strengthening should involve the added teaching of CT and/or MR imaging, as well as an expanded understanding of molecular medicine disciplines including molecular biology and pathology, proteomics or genomics.

5. Together we must address reimbursement issues and generate and promote proof of cost-efficacy of nuclear medicine imaging and therapy in clinical settings. This entails the appreciation and adoption of standardized imaging protocols, currently a major obstacle to the general adoption of dual modality imaging. Together with referring parties and users of standard imaging methods, we need to initiate cost-effectiveness studies that entail evaluation of data on the effect of nuclear medicine imaging on overall survival, quality of life and cost-effectiveness.

All five points represent the core of a "status go". If we start now we will be able to position and maintain nuclear medicine in the landscape of medical disciplines for the years to come. It will not be enough to think about these problems together and to talk about them together; we have to act together towards solutions to these problems. We need to remind ourselves of the merits of nuclear medicine: “... A chemist may think Physics, a physicist may think Chemistry, a botanist may think Chemistry and Physics, because all areas of expertise overlap ..." (Georg von Hevesy, 1885-1966) [19]. It is the multidisciplinary nature of our profession that made us strong, and it this strength that can help us grow. Let's move from "What can nuclear medicine do for us?" to "What can we do with nuclear medicine for others?". That's a "status go" to decay for.

\section{References}

1. Hoefnagel CA. Radionuclide therapy revisited. Eur J Nucl Med. 1991;18(6):408-31.

2. Schubiger PA, Alberto R, Smith A. Vehicles, chelators, and radionuclides: choosing the "building blocks" of an effective therapeutic radioimmunoconjugate. Bioconjug Chem. 1996;7(2):165-179.

3. Gnanasegaran G, Ballinger JR, Molecular Imaging - Novel tracers for SPECT/CT, Eur J Nucl Med Mol Imaging 2014 (Suppl 1), accepted.

4. Wahl RL, Quint LE, Cieslak RD, Aisen AM, Koeppe RA, Meyer CR. "Anatometabolic" tumor imaging: fusion of FDG PET with CT or MRI to localize foci of increased activity. J Nucl Med. 1993;34(7):1190-7.

5. Townsend DW. Multimodality imaging of structure and function. Phys Med Biol. 2008;53(4):R1-R39.

6. Czernin J, Allen-Auerbach M, Schelbert HR. Improvements in cancer staging with PET/CT: literature-based evidence as of September 2006. J Nucl Med. 2007;48 Suppl 1:78S-88S.

7. Kinahan PE, Hasegawa BH, Beyer T. X-ray-based attenuation correction for positron emission tomography/computed tomography scanners. Semin Nucl Med. 2003;33(3):166-79.

8. Chan C, Fulton R, Feng DD, Meikle S. Regularized image reconstruction with an anatomically adaptive prior for positron emission tomography. Phys Med Biol. 2009;54(24):7379-400.

9. Fritz SG, Shikhaliev PM. CZT detectors used in different irradiation geometries: simulations and experimental results. Med Phys. 2009;36(4):1098-108.

10. Weber WA. Is there evidence for evidence-based medical imaging? J Nucl Med. 2011;52 Suppl 2:74S-6S.

11. Wieder H, Freudenberg LS, Czernin J, Navar BN, Israel O, Beyer T. Variations of clinical SPECT/CT operations: an international survey. Nuklearmedizin. 2012;51(4):154-60.

12. Beyer T, Czernin J, Freudenberg LS. Variations in clinical PET/CT operations: results of an international survey of active PET/CT users. J Nucl Med. 2011;52(2):303-10.

13. Graham MM, Badawi RD, Wahl RL. Variations in PET/CT methodology for oncologic imaging at U.S. academic medical centers: an imaging response assessment team survey. J Nucl Med. 2011;52(2):311-7.

14. Catana C, Guimaraes AR, Rosen BR. PET and MR imaging: the odd couple or a match made in heaven? J Nucl Med. 2013;54(5):815-24.

15. Bailey DL, Barthel H, Beyer T, Boellaard R, Gückel B, Hellwig D, et al. Summary report of the first international workshop on PET/MR Imaging, March 19-23, 2012, Tübingen, Germany. Mol Imaging Biol. 2013;15(4):361-71.

16. Jadvar H, Colletti PM. Competitive advantage of PET/MRI. Eur J Radiol. 2013. doi:10.1016/j.ejrad.2013.05.028

17. Wester HJ. GMP production. Shaping the future. Nuklearmedizin. 2012;51(1):N1-4.

18. Schwaiger M, Wester HJ. How many PET tracers do we need? J Nucl Med. 2011;52 Suppl 2:36S-41S.

19. Niese S.: Georg von Hevesy: 1885-1966; Wissenschaftler ohne Grenzen. Münster/Westfalen: Principal-Verlag; 2009, ISBN 978-389969-081-1. 2009. 